\title{
Correlation of liver-to-spleen ratio on computed tomography with colorectal polyps
}

\author{
Yifei Zhou ${ }^{1 *}$, Miaofang Yang ${ }^{12^{*}}$, HaiJun Wan ${ }^{1}$, Yarong Yang ${ }^{2}$, Ying Qi' ${ }^{1}$ Fangyu Wang ${ }^{1}$ \\ 'Department of Gastroenterology and Hepatology, School of Medicine, Jinling Hospital, Nanjing University, Nanjing, Jiangsu, China \\ 2Department of Gastroenterology and Hepatology, Jinling Hospital, Southern Medical University, Nanjing, Jiangsu, China \\ *These authors are equally contributed to this paper.
}

\begin{abstract}
Aim of the study: To explore the correlation of the liver-to-spleen (L/S) ratio on computed tomography (CT) with colorectal polyps.

Material and methods: Consecutive participants from Jinling Hospital Affiliated to Nanjing University who underwent routine biochemical tests, colonoscopies, and CT between January 2018 and December 2019 were selected. The L/S ratio on CT was used to measure the liver fat content. Colonoscopy findings were applied to create the polyp-free group and colorectal polyp group. All included subjects were also classified in the non-alcoholic fatty liver disease (NAFLD) group or the non-NAFLD group according to the CT (L/S) ratio to identify risk factors for colorectal polyps. All data were analysed with SPSS 25 software.

Results: Among 481 participants, 27.8\% (79/284) of the patients were diagnosed with NAFLD in the colorectal group, which was higher than the corresponding proportion of the polyp-free group [9.1\% (18/197)]. In NAFLD patients, most adenomatous polyps were found in the transverse colon, and hyperplastic polyps were largely located in the rectum. Linear regression suggested that the $\mathrm{CT}(\mathrm{L} / \mathrm{S})$ ratio correlated with the number of colorectal polyps and with the number of adenomatous polyps. After adjusting for confounding factors, multivariate analysis indicated that NAFLD was an independent risk factor for adenomatous polyps and hyperplastic polyps.

Conclusions: A lower $\mathrm{CT}(\mathrm{L} / \mathrm{S})$ ratio (higher liver fat content) was significantly correlated with a higher risk of colorectal polyps. This finding suggested that NAFLD patients with a reduced CT (L/S) ratio need to undergo colonoscopy examinations to detect high-risk colorectal polyps in a timely manner.
\end{abstract}

Key words: liver fat content, colorectal polyps, non-alcoholic fatty liver disease, computed tomography, liverto-spleen ratio.

Address for correspondence

Fangyu Wang, Department of Gastroenterology and Hepatology, School of Medicine, Jinling Hospital, Nanjing University, Zhongshan East Road 305, Nanjing 210002 Jiangsu, China, phone: +86-025-80860151, +86-13515100636,

e-mail: wangfangyu65@126.com, wangfy65@nju.edu.cn

\section{Introduction}

According to the International Agency for Research on Cancer, Globocan 2018, colorectal cancer (CRC) is the third most commonly diagnosed cancer and the second leading cause of cancer death. The 5-year survival rates vary from $90 \%$ in the early stage to $14 \%$ in the advanced stage of the disease [1]. Currently, developing countries are at a stage of unprecedented indus- trialization, which leads to Western dietary patterns, obesity, and a sedentary lifestyle. As a result, the CRC incidence rates in these countries are increasing [2]. Colorectal adenomatous polyps (CAPs) and hyperplastic polyps (HPs) have been considered precursors of sporadic and hereditary colorectal cancer [3,4]. Moreover, patients with either 3 or more adenomas, high-grade dysplasia, villous features, or an adenoma $1 \mathrm{~cm}$ or larger are at high risk of progressing to CRC [5]. 
Therefore, the types and number of polyps play a crucial role in the pathogenesis of high-risk colorectal polyps, which are indeed worthy of attention.

Recently, several studies have demonstrated that non-alcoholic fatty liver disease (NAFLD) is associated with colorectal polyps and even CRC. The first cross-sectional study was conducted by Hwang et al. [6], who demonstrated that NAFLD was associated with an increased risk of CAP [odds ratio $(\mathrm{OR})=1.28$, 95\% confidential interval (CI): 1.03-1.60)]. Recent research by Cho et al. [7] found that biopsy-proven non-alcoholic steatohepatitis (NASH) was an independent risk factor for colorectal polyps $(\mathrm{OR}=2.08$, 95\% CI: 1.12-3.86) and advanced colorectal neoplasms $(\mathrm{OR}=2.81,95 \% \mathrm{CI}: 1.01-7.87)$. Liver fat content measurements are known to be significant for the diagnosis and monitoring of NAFLD. However, limited studies are available in the literature to evaluate the relevance of liver fat content and the number of colorectal polyps. Additionally, the contrasting relationship between two different types of colorectal polyps and NAFLD seems controversial. Therefore, we used the liver-tospleen ratio on computed tomography (CT) as a measure of liver fat content and investigated the relationship between the CT (L/S) ratio and colorectal polyps.

\section{Aim of the study}

Our study aims to explore the correlation of the L/S ratio on CT with colorectal polyps.

\section{Material and methods}

\section{Subjects}

Consecutive participants from our University Hospital who underwent routine biochemical tests, colonoscopies, and CT between January 2018 and December 2019 were selected. All relevant information was collected from medical records in the Electronic Medical Records System (EMRS) written by clinicians and was convincing and reliable; the collected data included smoking and alcohol consumption histories, underlying diseases, and family histories.

A total of 1077 participants were screened, of whom 596 were excluded because of a history of cancer $(n=175)$, inflammatory bowel disease $(n=226)$, autoimmune disease $(n=17)$, viral hepatitis $(n=102)$, alcohol abuse (males $>30 \mathrm{~g} /$ day, females $>20 \mathrm{~g} /$ day) [8] $(n=35)$, liver cirrhosis $(n=4)$, incomplete colonoscopies $(n=8)$, polypectomy within a year $(n=3)$, enterectomy histories $(n=5)$, Peutz-Jeghers syndrome $(n=3)$, schistosomiasis $(n=3)$, intestinal tuberculosis $(n=2)$, familial adenomatous polyposis $(n=2)$, multiple hepatic cysts $(n=2)$, pancreatitis $(n=2)$, a history of splenectomy $(n=2)$, anticoagulant therapies $(n=2)$, lack of pathology $(n=2)$ and intestinal obstruction $(n=1)$. As a result, 481 participants were included.

Anthropometric measurements and laboratory examinations:

Patient weight $(\mathrm{kg})$ and height $(\mathrm{m})$ were recorded by well-trained nurses. Body mass index (BMI) was calculated by weight $(\mathrm{kg})$ divided by height squared $\left(\mathrm{m}^{2}\right)$. Blood pressure was measured using an electronic sphygmomanometer (Omron, Kyoto, Japan). Venous blood samples were drawn from cubital veins after at least 12 hours of fasting, and plasma was separated by centrifugation within the stipulated time. All biochemistry results, such as plasma glucose and lipid metabolism, were measured by a Hitachi Automatic Analyzer B2400 (Hitachi, Tokyo, Japan).

\section{Colonoscopy}

Before the colonoscopy, all patients were given $4 \mathrm{~L}$ polyethylene glycol lavage solution for complete bowel preparation. Skilled gastroenterologists performed colonoscopy using a CF-H290 colonoscope (Olympus, Tokyo, Japan), and colonoscopy was completed when the ileocecal valve, caecum, and appendicular orifice were visualised. Polypoid lesions were biopsied or resected (endoscopic mucosal resection - EMR) for pathological diagnosis, and the location, size and number of lesions were recorded. If the number of polyps exceeded five, it was recorded as five in the analysis. According to the pathological results, the colorectal polyps were defined as CAPs and HPs. The location was classified as the ileocecal junction, ascending colon, transverse colon, descending colon, sigmoid colon and rectum. The size was estimated, and the number of lesions was counted by gastroenterologists.

\section{Diagnostic criteria}

Unenhanced CT of the liver was performed by a 64-section multidetector row scanner (SOMATOM Definition; Siemens Medical Solutions, Erlangen, Germany). The $\mathrm{L} / \mathrm{S}$ ratio on $\mathrm{CT}$ evaluated by unenhanced CT represented the liver fat content [9]. Moreover, patients with a $\mathrm{CT}(\mathrm{L} / \mathrm{S})$ ratio $<1$ were diagnosed with fatty liver [10]. The diagnostic criteria of diabetes mellitus were fasting plasma glucose (FPG) levels $\geq 7.0 \mathrm{mmol} / \mathrm{l}$, a random plasma glucose level $\geq 11.1 \mathrm{mmol} / \mathrm{l}$ and/or the current use of antidiabetic medications [11]. Coronary heart disease was diagnosed by CT angiography and/or by previous medical histories. Hypertension 
was defined as three measurements of a systolic blood pressure (SBP) $\geq 140 \mathrm{mmHg}$ and diastolic blood pressure $(\mathrm{DBP}) \geq 90 \mathrm{mmHg}$ in a day during the resting state and/or the current use of antihypertensive drugs.

\section{Statistical methods}

Statistical analysis was conducted using SPSS Statistics software version 25 (IBM Corporation, Armonk, New York). Demographic results are presented as the mean \pm standard deviation (SD) or percentages (\%) and were compared using the independent $t$-test or Kruskal-Wallis test for continuous variables and $\chi^{2}$ test for categorical variables. Linear regression was carried out to determine the relationship between colorectal polyps and the CT (L/S) ratio. ANOVA and Tukey's multiple comparisons test were used to analyse the location of different types of colorectal polyps. Binary logistic re- gression analysis was used to analyse the relationship between different types of colorectal polyps and other risk factors. Multivariate logistic regression analysis without interactions was performed to identify independent risk factors for colorectal polyps after adjusting for variables including age, sex, smoking history, hypertension, diabetes mellitus, NAFLD and the results of the binary logistic analysis. A two-sided $\mathrm{P}$ value less than 0.05 was considered statistically significant. Each OR is presented together with its $95 \%$ CI. All statistical charts were drawn by GraphPad Prism software version 8.0.2.

\section{Results}

\section{Subject characteristics and CT results}

The baseline characteristics and biochemical parameters of the study cohort are presented in Table 1

Table 1. Clinical and biochemical characteristics of the 481 subjects

\begin{tabular}{|c|c|c|c|c|c|c|c|}
\hline Characteristic & $\begin{array}{c}\text { Polyp-free } \\
n=197\end{array}$ & $\begin{array}{c}\text { Colorectal } \\
\text { polyps } \\
n=284\end{array}$ & $\begin{array}{c}\text { Adenomatous } \\
\text { polyps } \\
n=206\end{array}$ & $\begin{array}{c}\text { Hyperplastic } \\
\text { polyps } \\
n=168\end{array}$ & $\begin{array}{l}\text { P Polyp-free vs. } \\
\text { colorectal } \\
\text { polyps }\end{array}$ & $\begin{array}{l}\text { P Polyp-free vs. } \\
\text { adenomatous } \\
\text { polyps }\end{array}$ & $\begin{array}{l}\text { P Polyp-free vs. } \\
\text { hyperplastic } \\
\text { polyps }\end{array}$ \\
\hline Age (years) & $52.51 \pm 11.06$ & $55.92 \pm 10.38$ & $57.05 \pm 9.97$ & $55.08 \pm 10.82$ & $<0.05$ & $<0.05$ & 0.026 \\
\hline Sex (male), $n(\%)$ & $113(57.4)$ & $211(74.3)$ & $150(72.8)$ & $134(79.8)$ & $<0.05$ & 0.12 & $<0.05$ \\
\hline Diabetes mellitus, $n(\%)$ & $26(13.2)$ & $51(18.0)$ & $35(17)$ & $33(19.6)$ & 0.16 & 0.57 & 0.13 \\
\hline Coronary heart disease, $n(\%)$ & $14(7.1)$ & $24(8.5)$ & $18(8.7)$ & $16(9.5)$ & 0.51 & 0.94 & 0.62 \\
\hline Hypertension, $n(\%)$ & $40(20.3)$ & $101(35.6)$ & $75(36.4)$ & $64(38.1)$ & $<0.05$ & 0.02 & 0.03 \\
\hline Smoking, $n(\%)$ & $39(19.8)$ & $91(32)$ & $64(31.1)$ & $58(34.5)$ & $<0.05$ & 0.08 & 0.01 \\
\hline $\mathrm{SBP}(\mathrm{mmHg})$ & $124.44 \pm 14.03$ & $126.67 \pm 12.46$ & $125.84 \pm 11.71$ & $128.09 \pm 13.05$ & 0.07 & 0.28 & 0.01 \\
\hline $\mathrm{DBP}(\mathrm{mmHg})$ & $77.64 \pm 10.95$ & $79.50 \pm 10.07$ & $79.37 \pm 9.87$ & $80.57 \pm 10.58$ & 0.06 & 0.10 & 0.01 \\
\hline $\mathrm{BMI}\left(\mathrm{kg} / \mathrm{m}^{2}\right)$ & $24.41 \pm 3.22$ & $24.69 \pm 2.98$ & $24.61 \pm 3.10$ & $25.17 \pm 2.94$ & 0.33 & 0.53 & 0.02 \\
\hline $\mathrm{HDL}(\mathrm{mmol} / \mathrm{l})$ & $1.18 \pm 0.23$ & $1.21 \pm 0.86$ & $1.25 \pm 0.99$ & $1.13 \pm 0.24$ & 0.63 & 0.33 & 0.04 \\
\hline LDL (mmol/l) & $2.76 \pm 0.66$ & $2.87 \pm 1.12$ & $2.88 \pm 1.24$ & $2.78 \pm 0.70$ & 0.22 & 0.23 & 0.78 \\
\hline Total cholesterol (mmol/l) & $4.52 \pm 1.47$ & $4.57 \pm 1.11$ & $4.57 \pm 1.18$ & $4.47 \pm 0.88$ & 0.67 & 0.71 & 0.70 \\
\hline Triglycerides (mmol/l) & $1.92 \pm 2.40$ & $2.16 \pm 2.05$ & $2.13 \pm 2.14$ & $2.03 \pm 1.43$ & 0.24 & 0.35 & 0.60 \\
\hline FPG (mmol/l) & $5.33 \pm 1.37$ & $5.45 \pm 1.50$ & $5.42 \pm 1.49$ & $5.55 \pm 1.65$ & 0.37 & 0.53 & 0.16 \\
\hline Albumin (g/l) & $41.36 \pm 4.02$ & $42.04 \pm 3.49$ & $41.91 \pm 3.43$ & $42.19 \pm 3.47$ & 0.049 & 0.14 & 0.04 \\
\hline AST (IU/I) & $20.51 \pm 12.38$ & $20.43 \pm 8.02$ & $20.65 \pm 8.60$ & $19.71 \pm 6.05$ & 0.93 & 0.89 & 0.45 \\
\hline ALT (IU/I) & $22.49 \pm 18.15$ & $24.10 \pm 14.55$ & $23.77 \pm 14.45$ & $24.23 \pm 14.20$ & 0.28 & 0.43 & 0.31 \\
\hline GGT (IU/I) & $29.06 \pm 25.05$ & $32.88 \pm 34.05$ & $32.57 \pm 35.59$ & $30.54 \pm 24.55$ & 0.18 & 0.25 & 0.57 \\
\hline Total bilirubin $(\mu \mathrm{mol} / \mathrm{l})$ & $10.56 \pm 5.34$ & $10.65 \pm 5.00$ & $10.82 \pm 5.30$ & $10.58 \pm 4.85$ & 0.85 & 0.61 & 0.59 \\
\hline Platelets $\left(\times 10^{3} / \mu \mathrm{l}\right)$ & $214.32 \pm 56.22$ & $205.46 \pm 57.06$ & $202.49 \pm 57.75$ & $209.44 \pm 63.45$ & 0.09 & 0.04 & 0.44 \\
\hline PT (s) & $11.48 \pm 0.69$ & $11.31 \pm 0.78$ & $11.24 \pm 0.68$ & $11.34 \pm 0.83$ & 0.01 & $<0.05$ & 0.08 \\
\hline $\mathrm{CT}(\mathrm{L} / \mathrm{S})$ & $1.20 \pm 0.20$ & $1.11 \pm 0.20$ & $1.11 \pm 0.19$ & $1.11 \pm 0.20$ & $<0.05$ & $<0.05$ & $<0.05$ \\
\hline
\end{tabular}

Bold values are statistically significant.

NAFLD - non-alcoholic fatty liver disease, SBP - systolic blood pressure, DBP - diastolic blood pressure, BMI - body mass index, HDL - high-density lipoprotein, LDL - low-density lipoprotein, FPG - fasting plasma glucose, AST - aspartate aminotransferase, ALT - alanine aminotransferase, GGT - $\gamma$-glutamyl transferase, PT - prothrombin time. The data are expressed as the means \pm standard deviations or $n(\%)$. 


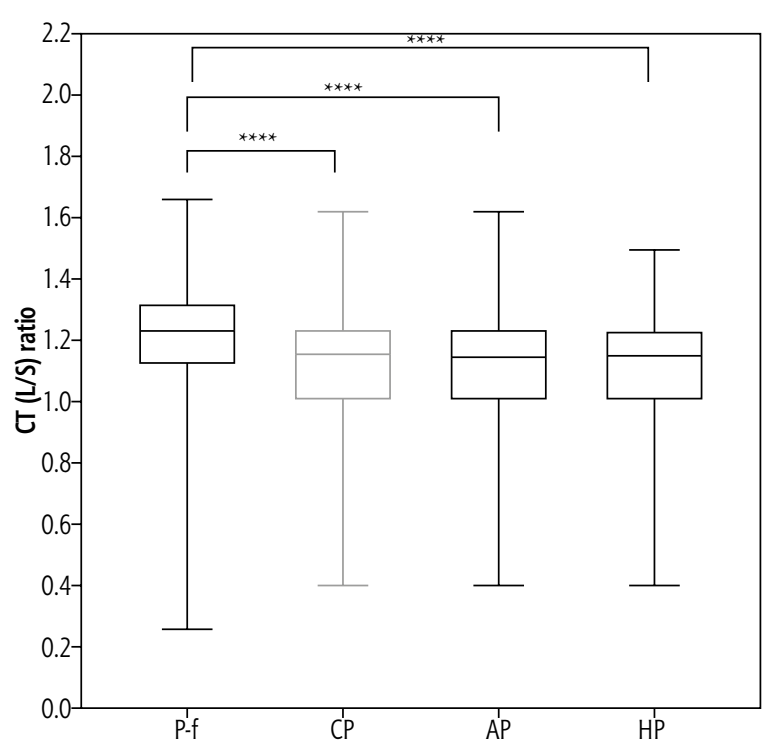

$P$-f - polyp-free, CP - colorectal polyp, AP - adenomatous polyp, HP - hyperplastic polyp Plot: Max to min.

Fig. 1. CT (L/S) ratio in patients with two different types of polyps. The CT (L/S) ratio was significantly lower in patients with adenomatous polyps and hyperplastic polyps compared to that in polyp-free patients

Table 2. Colonoscopy findings of patients with and without non-alcoholic fatty liver disease (NAFLD)

\begin{tabular}{lcccc}
\hline NAFLD & $\begin{array}{c}\text { Polyp-free } \\
\boldsymbol{n}(\%)\end{array}$ & $\begin{array}{c}\text { Colorectal } \\
\text { polyps } \\
\boldsymbol{n}(\%)\end{array}$ & $\begin{array}{c}\text { Total } \\
\boldsymbol{N}(\%)\end{array}$ & $\boldsymbol{P}$ \\
\hline $\begin{array}{l}\text { Yes [CT }(\mathrm{L} / \mathrm{S})<1] \\
n(\%)\end{array}$ & $18(9.1)$ & $79(27.8)$ & $97(20.2)$ & $<0.05$ \\
\hline $\begin{array}{l}\text { No }[C T(L / S) \geqslant 1] \\
n(\%)\end{array}$ & $179(90.9)$ & $205(72.2)$ & $384(79.8)$ & \\
\hline $\begin{array}{l}\text { Total } \\
N(\%)\end{array}$ & $197(100.0)$ & 284 & $481(100)$ & \\
\hline NAFLD - non-alcoholic fatty liver disease & & & & \\
\hline
\end{tabular}

Table 3. Location of the colorectal polyps in patients with non-alcoholic fatty liver disease (NAFLD)

\begin{tabular}{lccc}
\hline Location & $\begin{array}{c}\text { Adenomatous } \\
\text { polyps }(\boldsymbol{n})\end{array}$ & $\begin{array}{c}\text { Hyperplastic } \\
\text { polyps }(\boldsymbol{n})\end{array}$ & $\boldsymbol{P}$ \\
\hline Ileocecal junction & $0.03 \pm 0.18$ & $0.02 \pm 0.14$ & $<0.05$ \\
\hline Ascending colon & $0.19 \pm 0.43$ & $0.08 \pm 0.28$ & \\
\hline Transverse colon & $0.58 \pm 0.65$ & $0.31 \pm 0.55$ & \\
\hline Descending colon & $0.42 \pm 0.68$ & $0.24 \pm 0.48$ & \\
\hline Sigmoid colon & $0.29 \pm 0.49$ & $0.51 \pm 0.82$ & \\
\hline Rectum & $0.20 \pm 0.45$ & $0.63 \pm 0.91$ & \\
\hline
\end{tabular}

$n$ - number of the polyps

and Figure 1. The L/S ratio was lower in subjects with colorectal polyps, adenomatous polyps, and HPs than in those without polyps $(1.11 \pm 0.20,1.12 \pm 0.19$, and $1.12 \pm 0.20$ vs. $1.20 \pm 0.20, p<0.05)$. Significant differ- ences in age, sex (mostly male), hypertension, smoking history, albumin, and prothrombin time (PT) were found between participants without polyps and those with colorectal polyps $(p<0.05)$. Overall, patients with adenomatous polyps were older and more likely to have hypertension, a smoking history, a lower platelet count, and a lower PT. Moreover, patients with HPs were mostly male, older, and more likely to have hypertension, a smoking history, higher SBP, higher DBP, higher BMI, higher albumin levels, and lower high-density lipoprotein (HDL) levels.

\section{Colonoscopy results}

In the colorectal polyps group, $27.8 \%(79 / 284)$ of patients were diagnosed with NAFLD [CT $(\mathrm{L} / \mathrm{S})<1]$, which was higher than the corresponding proportion of the polyp-free group [9.1\% (18/197)] (see Table 2, $p<0.05)$. Moreover, in NAFLD patients, the adenomatous polyps were mostly found in the transverse colon, and HPs were mostly located in the rectum, which as a significant difference in location (see Table 3 and Fig. $2, p<0.05)$.

\section{Correlation of CT (L/S) ratio with colorectal polyps}

Linear regression suggested that the $\mathrm{CT}(\mathrm{L} / \mathrm{S})$ ratio correlates with the number of colorectal polyps (slope $=-1.408,95 \%$ CI: -2.072 to $-0.7441, p<0.05$; see Fig. 3) and adenomatous polyps (slope $=-0.6938$, $95 \%$ CI: -1.159 to -0.2282 , $p<0.05$; see Fig. 3 ). The results indicated that a lower CT (L/S) ratio corresponds to an increase in the number of colorectal polyps. The risk factors for colorectal polyps, such as NAFLD and other metabolic disorders, are presented in Table 4. In adenomatous polyp patients, univariate analysis suggested that NAFLD (OR $=2.50,95 \% \mathrm{CI}$ : 1.586-3.952, $p<0.05)$, age, sex, hypertension, platelet count, and PT were risk factors in binary logistic regression analysis. After adjusting for age, sex, diabetes mellitus, smoking, and PT, the multivariate analysis suggested that NAFLD (OR $=2.53,95 \%$ CI: $1.538-4.144, p<0.05)$ was an independent risk factor for adenomatous polyps. In HP patients, the univariate analysis demonstrated that NAFLD (OR $=2.27,95 \%$ CI: 1.445-3.576, $p<0.05$ ), sex, hypertension, smoking, and BMI were risk factors, and HDL was a protective factor. After adjusting for age, sex, diabetes mellitus, hypertension, smoking, BMI, and HDL, NAFLD (OR = 1.77, 95\% CI: 1.086$2.885, p=0.022$ ) was found to be an independent risk factor for HPs. 

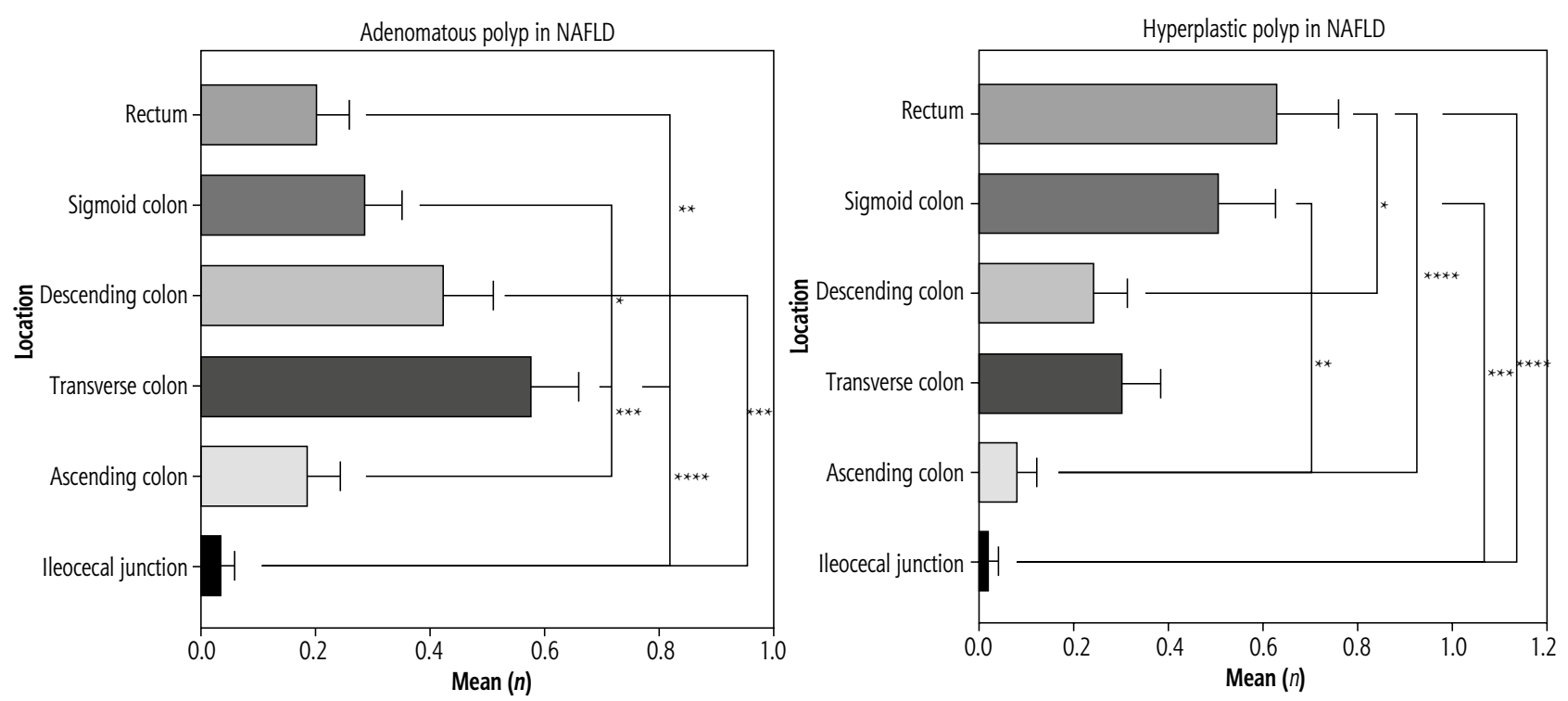

Fig. 2. Location of colorectal polyps in patients with non-alcoholic fatty liver disease (NAFLD). Most of the adenomatous polyps were found in the transverse colon, and hyperplastic polyps were largely located at the rectum, which meant that adenomatous polyps tended to develop at the proximal colon and hyperplastic polyps tended to develop at the distal colon
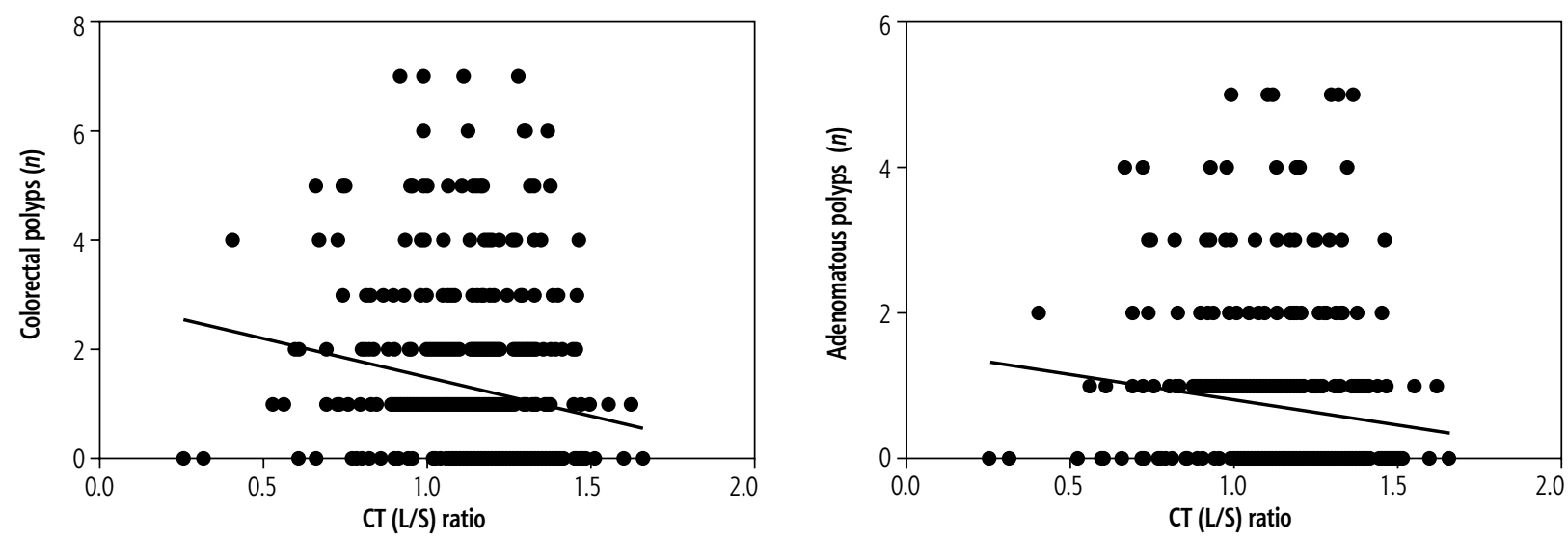

Fig. 3. Linear regression between the number of colorectal polyps and CT (L/S) ratio. The CT (L/S) ratio correlated with the number of colorectal polyps and adenomatous polyps, which meant that lower liver fat content correlated with a higher number of colorectal polyps

\section{Discussion}

The results from this study provide evidence that a lower CT (L/S) ratio was correlated with the number of colorectal polyps. After adjusting for confounding factors, including age, sex, glucose metabolism, and lipid metabolism, NAFLD was shown to be an independent risk factor for adenomatous polyps $(\mathrm{OR}=2.53$, 95\% CI: $1.538-4.144, p<0.05)$ and HPs (OR $=1.77$, 95\% CI: $1.086-2.885, p=0.022$ ). These findings suggested that NAFLD patients with higher liver fat content had a higher probability of having more adenomatous polyps than those with a normal liver fat content. High-risk colorectal polyps were defined by the presence of 3 or more adenomas, high-grade dysplasia, villous features, or an adenoma $1 \mathrm{~cm} \mathrm{[5]} \mathrm{or} \mathrm{larg-}$ er and had a high risk for progressing to CRC. Therefore, the type and number of polyps played an essential role in the pathogenesis of high-risk colorectal polyps, and NAFLD patients with high liver fat content had a tendency to develop such polyps, which are indeed worthy of attention.

The liver fat content can be measured by histology, ultrasound (US), CT, magnetic resonance imaging (MRI), and proton magnetic resonance spectroscopy ( $\left.{ }^{1} \mathrm{H}-\mathrm{MRS}\right)$, but histology is the most precise method. However, histology is an invasive process, and its clinical application is limited. Additionally, a biopsy could lead to sampling error. One previous study [12] of NAFLD patients found that the prevalence of colorectal adenomas was higher in NAFLD patients than in those without NAFLD (34.7\% vs. $21.5 \%, p=0.043)$. 
Table 4. Risk factors for colorectal polyps

\begin{tabular}{|c|c|c|c|c|c|c|c|c|c|c|c|c|}
\hline \multirow[t]{3}{*}{ Variable } & \multicolumn{6}{|c|}{ Adenomatous polyps } & \multicolumn{6}{|c|}{ Hyperplastic polyps } \\
\hline & \multicolumn{3}{|c|}{ Unadjusted } & \multicolumn{3}{|c|}{ Adjusted } & \multicolumn{3}{|c|}{ Unadjusted } & \multicolumn{3}{|c|}{ Adjusted } \\
\hline & OR & $95 \% \mathrm{Cl}$ & $p$ & OR & $95 \% \mathrm{Cl}$ & $p$ & OR & $95 \% \mathrm{Cl}$ & $p$ & OR & $95 \% \mathrm{Cl}$ & $p$ \\
\hline NAFLD & 2.50 & $1.586-3.952$ & $<0.05$ & 2.53 & $1.538-4.144$ & $<0.05$ & 2.27 & $1.445-3.576$ & $<0.05$ & 1.77 & $1.086-2.885$ & 0.022 \\
\hline Age & 1.04 & $1.022-10.59$ & $<0.05$ & 1.05 & $1.030-1.072$ & $<0.05$ & 1.01 & $0.990-1.025$ & 0.404 & 1.02 & $0.992-1.031$ & 0.245 \\
\hline Sex (male) & 1.56 & $1.050-2.303$ & 0.028 & 1.33 & $0.841-2.109$ & 0.222 & 2.55 & $1.644-3.959$ & $<0.05$ & 1.82 & $1.111-2.991$ & 0.017 \\
\hline Diabetes mellitus & 1.15 & $0.696-1.854$ & 0.611 & 0.62 & $0.359-1.080$ & 0.091 & 1.49 & $0.910-2.455$ & 0.113 & 0.98 & $0.570-1.689$ & 0.946 \\
\hline $\begin{array}{l}\text { Coronary heart } \\
\text { disease }\end{array}$ & 1.22 & $0.628-2.372$ & 0.556 & & & & 1.39 & $0.710-2.730$ & 0.335 & & & \\
\hline Hypertension & 1.81 & $1.220-2.695$ & 0.003 & 1.38 & $0.886-2.151$ & 0.154 & 1.89 & $1.259-2.825$ & 0.02 & 1.63 & $1.042-2.535$ & 0.032 \\
\hline Smoking & 1.43 & $0.952-2.139$ & 0.085 & 1.40 & $0.876-2.227$ & 0.161 & 1.76 & $1.168-2.667$ & 0.007 & 1.34 & $0.846-2.122$ & 0.213 \\
\hline BMI & 1.01 & $0.950-1.068$ & 0.814 & & & & 1.10 & $1.037-1.174$ & 0.002 & 1.05 & $0.981-1.126$ & 0.160 \\
\hline HDL & 1.48 & $0.741-2.961$ & 0.267 & & & & 0.39 & $0.174-0.864$ & 0.021 & 0.721 & $0.318-1.634$ & 0.433 \\
\hline LDL & 1.13 & $0.912-1.389$ & 0.271 & & & & 0.92 & $0.732-1.152$ & 0.462 & & & \\
\hline Total cholesterol & 1.02 & $0.883-1.172$ & 0.815 & & & & 0.91 & $0.768-1.084$ & 0.298 & & & \\
\hline Triglycerides & 1.02 & $0.941-1.108$ & 0.612 & & & & 0.99 & $0.902-1.076$ & 0.738 & & & \\
\hline FPG & 1.02 & $0.899-1.152$ & 0.785 & & & & 1.11 & $0.977-1.256$ & 0.111 & & & \\
\hline Platelets & 0.99 & $0.993-1$ & 0.029 & & & & 1.00 & $0.997-1.003$ & 0.921 & & & \\
\hline PT & 0.61 & $0.465-0.805$ & $<0.05$ & 0.619 & $0.462-0.829$ & 0.001 & 0.89 & $0.694-1.158$ & 0.401 & & & \\
\hline
\end{tabular}

Bold values are statistically significant. NAFLD was an independent risk factor for adenomatous polyps and hyperplastic polyps.

OR - odds ratio, 95\% CI - 95\% confidential interval, NAFLD - non-alcoholic fatty liver disease, SBP - systolic blood pressure, DBP - diastolic blood pressure, BMI - body mass index, FPG - fasting plasma glucose, PT - prothrombin time

Similarly, among biopsy-proven NASH patients, the incidence of adenomas was higher in NASH patients than in those with simple steatosis $(51.0 \%$ vs. $25.6 \%$, $p=0.005$ ). Another study by Cho et al. [7] revealed that biopsy-proven NASH was an independent risk factor for colorectal polyps $(\mathrm{OR}=2.08,95 \%$ CI: 1.12-3.86) and advanced colorectal neoplasms $(\mathrm{OR}=2.81,95 \% \mathrm{CI}$ : 1.01-7.87), which was similar to our study. US is a non-invasive, low-cost method that is accessible in most clinics; however, it has several limitations, such as operator and equipment dependency [13], and is only a qualitative assessment of the fatty liver. MRI and ${ }^{1} \mathrm{H}$-MRS have been proven to be accurate non-invasive methods, and ${ }^{1} \mathrm{H}-\mathrm{MRS}$ has even been regarded as the gold standard method to assess hepatic lipid content [14]. However, the technical requirements of ${ }^{1} \mathrm{H}-\mathrm{MRS}$ remain high, and it is hardly accessible in most clinics. CT is acceptable, moderately cost-effective, objective, and widely used as a health examination and to diagnose diseases. Non-contrast CT was demonstrated to have the best correlation with MRS, and ultrasound shear-wave elastography showed a very poor correlation with MRS, which means that non-contrast CT can serve as an accurate and non-invasive biomarker for identifying fatty liver disease through the quantifica- tion of steatosis [15]. Eventually, the CT (L/S) ratio to measure liver fat content was found to be more appropriate, non-invasive, cost-effective and objective than US and MR [9]. Additionally, hepatic iron deposition did not seem to affect the application of CT (L/S) in diagnosing NAFLD [16]. Aktas et al. [17] found that the liver density was lower in CRC patients than in those without CRC on contrast-enhanced CT. Our study showed that the liver density was lower in patients who had more adenomatous polyps than in those with a normal liver density, as measured by unenhanced CT. Most CRC develops through the adenoma-carcinoma sequence[18]. As the liver fat content was easily accessible, we suggest that NAFLD patients with high liver fat content undergo colonoscopy in a timely manner to detect high-risk colorectal polyps.

Patients with adenomatous polyps were older and were more likely to have hypertension, a smoking history, lower platelet count, and lower PT. We speculated that the older the patients were, the higher was the incidence of adenomatous polyps. Hypertension and smoking history have already been regarded as risk factors for colorectal polyps [19]. Platelet count and PT were associated with liver function, which suggested that patients with adenomatous polyps may have 
hepatic dysfunction. The HP group was mostly male and was more likely to have hypertension, a smoking history, higher SBP, higher DBP, higher BMI, and lower HDL. This result suggested that males were more likely to develop HPs than females. A higher BMI and lower HDL may imply a lipometabolic disturbance in patients with HPs.

In NAFLD patients, most adenomatous polyps were found in the transverse colon, and HPs were largely located in the rectum. This result indicated that adenomatous polyps tended to develop at the proximal colon and HPs tended to develop at the distal colon. This was also found in the cohort of Cho et al. [7], who discovered that both low-grade CAPs and advanced colorectal neoplasms were mostly located in the ascending colon, as well as in the study by Bhatt et al. [20]. We hypothesized that this finding may be related to the gut-liver axis [21]. Venous drainage of the proximal colon almost returns to the liver through the hepatic portal vein with two branches called the superior and inferior mesenteric veins. Venous drainage of the distal colon, however, partly returns to the liver through the portal vein and partly returns to the inferior vena cava [22]. Low-grade inflammation in the liver interacts with the intestinal mucosa in the gut, especially in the proximal colon, and creates a suitable environment for the development of high-risk polyps, and this point deserves further study.

There were several limitations of our study. First, this was a single-centre, retrospective study that failed to determine the causality between colorectal polyps and high liver fat content. Therefore, we need a large, multicentre, prospective study to clarify the causality between colorectal polyps and high liver fat content. Second, experiments will be carried out to explore the underlying mechanism in the future. Third, insulin, insulin resistance (IR) and insulin-like growth factor-1 (IGF-1) were not evaluated due to the retrospective nature of our study, because measurements of insulin and IGF-1 levels are not routine test items before colonoscopy. It has been reported that IR and increased IGF-1 levels act as cellular proliferation and precancerous factors, which can lead to colorectal cancer and polyp development in NAFLD patients [23], and we hope that there will be further studies on the relationship between IR and colorectal polyps in NAFLD patients in the future, as this would be quite fascinating.

In conclusion, our study provided evidence that a lower CT (L/S) ratio (higher liver fat content) was significantly associated with a higher risk of colorectal polyps. Moreover, NAFLD was an independent risk factor for adenomatous polyps, which could be a precursor of CRC. Therefore, NAFLD patients with increased liver fat content had an increased tendency to develop adenomatous polyps and CRC, which are indeed worthy of attention. We suggest that NAFLD patients with high liver fat content undergo timely colonoscopy and have shortened follow-up periods after undergoing EMR for colorectal polyps. We also advise clinicians to pay attention to the transverse and rectal colon when performing colonoscopy in NAFLD patients with high liver content to improve the detection of colorectal polyps.

\section{Ethics approval}

All procedures performed in studies involving human participants were in accordance with the ethical standards of the institutional and/or national research committee and with the 1964 Helsinki Declaration and its later amendments or comparable ethical standards. This study was approved by the Institutional Review Board of Jinling Hospital (2014NZKY-007-01).

\section{Acknowledgements}

The authors appreciate all the participants and clinicians in Jinling Hospital for their support.

\section{Funding}

This study was funded by the National Natural Science Foundation of China, grant number no. 81873559.

\section{Disclosure}

The authors declare no conflict of interest.

\section{References}

1. Schreuders EH, Ruco A, Rabeneck L, et al. Colorectal cancer screening: a global overview of existing programmes. Gut 2015; 64: 1637-1649.

2. Keum N, Giovannucci E. Global burden of colorectal cancer: emerging trends, risk factors and prevention strategies. Nat Rev Gastroenterol Hepatol 2019; 16: 713-732.

3. Cannon-Albright LA, Skolnick MH, Bishop DT, et al. Common inheritance of susceptibility to colonic adenomatous polyps and associated colorectal cancers. N Engl J Med 1988; 319: 533-537.

4. Hawkins NJ, Ward RL. Sporadic colorectal cancers with microsatellite instability and their possible origin in hyperplastic polyps and serrated adenomas. J Natl Cancer Inst 2001; 93: 13071313.

5. Winawer SJ, Zauber AG, Fletcher RH, et al. Guidelines for colonoscopy surveillance after polypectomy: a consensus update by the US Multi-Society Task Force on Colorectal Cancer and the American Cancer Society. Gastroenterology 2006; 130: 18721885 . 
6. Hwang ST, Cho YK, Park JH, et al. Relationship of non-alcoholic fatty liver disease to colorectal adenomatous polyps. J Gastroenterol Hepatol 2010; 25: 562-567.

7. Cho Y, Lim SK, Joo SK, et al. Nonalcoholic steatohepatitis is associated with a higher risk of advanced colorectal neoplasm. Liver Int 2019; 39: 1722-1731.

8. Chalasani N, Younossi Z, Lavine JE, et al. The diagnosis and management of non-alcoholic fatty liver disease: practice guideline by the American Gastroenterological Association, American Association for the Study of Liver Diseases, and American College of Gastroenterology. Gastroenterology 2012; 142: 15921609.

9. Mehta SR, Thomas EL, Bell JD, et al. Non-invasive means of measuring hepatic fat content. World J Gastroenterol 2008; 14: 3476-3483.

10. Park YS, Park SH, Lee SS, et al. Biopsy-proven nonsteatotic liver in adults: estimation of reference range for difference in attenuation between the liver and the spleen at nonenhanced CT. Radiology 2011; 258: 760-766.

11. American Diabetes A. Diagnosis and classification of diabetes mellitus. Diabetes Care 2014; 37 Suppl: S81-S90.

12. Wong VW, Wong GL, Tsang SW, et al. High prevalence of colorectal neoplasm in patients with non-alcoholic steatohepatitis. Gut 2011; 60: 829-836.

13. Mishra P, Younossi ZM. Abdominal ultrasound for diagnosis of nonalcoholic fatty liver disease (NAFLD). Am J Gastroenterol 2007; 102: 2716-2717.

14. Hwang JH, Choi CS. Use of in vivo magnetic resonance spectroscopy for studying metabolic diseases. Exp Mol Med 2015; 47: e139.

15. Graffy PM, Pickhardt PJ. Quantification of hepatic and visceral fat by CT and MR imaging: relevance to the obesity epidemic, metabolic syndrome and NAFLD. Br J Radiol 2016; 89: 2015 1024.

16. Yang SP, Park SH, Lee SS, et al. Biopsy-proven nonsteatotic liver in adults: Estimation of reference range for difference in attenuation between the liver and the spleen at nonenhanced CT. Radiology 2011; 258: 760-766.

17. Aktas E, Uzman M, Yildirim O, et al. Assessment of hepatic steatosis on contrast enhanced computed tomography in patients with colorectal cancer. Int J Clin Exp Med 2014; 7: 4342-4346.

18. Parizadeh SM, Parizadeh SA, Alizade-Noghani M, et al. Association between non-alcoholic fatty liver disease and colorectal cancer. Expert Rev Gastroenterol Hepatol 2019; 13: 633-641.

19. Pan J, Cen L, Xu L, et al. Prevalence and risk factors for colorectal polyps in a Chinese population: a retrospective study. Sci Rep 2020; 10: 6974.

20. Bhatt BD, Lukose T, Siegel AB, et al. Increased risk of colorectal polyps in patients with non-alcoholic fatty liver disease undergoing liver transplant evaluation. J Gastrointest Oncol 2015; 6: 459-468.

21. Kirpich IA, Marsano LS, McClain CJ. Gut-liver axis, nutrition, and non-alcoholic fatty liver disease. Clin Biochem 2015; 48: 923-930.

22. THIEME Atlas of anatomy, 3 volume Hardcover Set. Thieme 2007.

23. Alam S, Mustafa G, Alam M, et al. Insulin resistance in development and progression of nonalcoholic fatty liver disease. World J Gastrointest Pathophysiol 2016; 7: 211-217. 